\title{
ENFOQUES DE PROGRAMAS PARA LA INCLUSIÓN LABORAL DE LOS JÓVENES POBRES: LO INSTITUCIONAL COMO SOPORTE SUBJETIVO
}

\author{
CLAUDiA JACINTO* \\ VERÓNICA MILLENAAR $^{* *}$
}

\begin{abstract}
RESUMEN
Los problemas de empleo y de desafiliación de los jóvenes se han tornado en un verdadero desafío para las políticas públicas y acciones de la sociedad civil. Desde hace tiempo, se implementan programas y estrategias diversas que, en gran medida, comparten un diagnóstico inespecífico sobre el trasfondo de los problemas en el mercado de trabajo y los llamados «déficits» de formación. Estos enfoques resultan limitados frente a la compleja trama de dimensiones estructurales, institucionales, familiares, individuales y subjetivas que colocan a los jóvenes, en particular a los más pobres, en situación de desventaja. El presente artículo se plantea discutir en particular uno de estos aspectos: el nivel institucional; para reflexionar sobre las formas en que las instituciones intentan intervenir en la transición juvenil, frente a los procesos de individuali ${ }^{2} \quad$ ón y de debilitamiento institucional. De este modo, se han seleccionado dos casos de organizaciones en Argentina que se proponen generar un fuerte impacto sobre las subjetividades de los jóvenes, a través de abordajes que toman en cuenta las «constelaciones de desventajas» juveniles, de un modo amplio. Este análisis de dos instituciones que dan lugar preferencial a los abordajes subjetivos, abre la reflexión acerca de las posibilidades institucionales de proveer nuevos soportes colectivos y contribuir a la individualización de los sujetos y al desarrollo de sus propias estrategias.
\end{abstract}

\section{PALABRAS CLAVE: TRAYECTORIAS DE INSERCIÓN LABORAL, JUVENTUD, INSTITUCIONES}

* Doctora en Sociología de la Universidad de París III, investigadora del CONICET de Argentina y coordinadora de REDETIS y Programa de Juventud, Educación y Trabajo (PREJET) del IDES.

E-Mail: cjacinto@redetis.iipe-ides.org.ar.

** Socióloga de la Universidad de Buenos Aires (UBA). Realiza su Maestría en Ciencias Sociales en el IDES-UNGS.

E-Mail: veronicamillenaar@argentina.com.

Este artículo recoge algunos resultados parciales del proyecto «Trayectorias educativo-laborales de jóvenes. Incidencia de políticas y programas de inclusión social» que cuenta con el apoyo de la Agencia Nacional de Investigaciones Científicas y Técnicas de la Argentina. 


\title{
ENFOQUES DE PROGRAMAS PARA A INCLUSÃO TRABALHISTA DE JOVENS POBRES: O INSTITUCIONAL COMO SUPORTE SUBJETIVO
}

\begin{abstract}
RESUMO
Os problemas de emprego e de desassociação dos jovens têm se tornado um verdadeiro desafio para as políticas públicas e ações da sociedade civil. Há muito têm-se implementado programas e diferentes estratégias que, em grande parte, compartilham um diagnóstico pouco específico sobre o que está além dos problemas no mercado de trabalho e dos chamados «déficits» de formação. Estes enfoques mostram-se limitados frente à complexa trama de dimensões estruturais, institucionais, familiares, individuais e subjetivas que colocam os jovens, em particular os mais pobres, em situação de desvantagem. O presente artigo propõe-se a discutir particularmente o nível institucional, com o objetivo de refletir sobre as formas pelas quais as instituições tentam intervir na transição juvenil, frente aos processos de individualização e de enfraquecimento institucional. Deste modo, foram selecionados dois casos de organizações na Argentina que se propõem a exercer um forte impacto sobre as subjetividades dos jovens, através de abordagens que consideram as «constelações de desvantagens» juvenis de modo amplo. Esta análise de duas instituições que privilegiam as abordagens subjetivas apresenta uma reflexão sobre as possibilidades institucionais em fornecer novos suportes coletivos, contribuindo com a individualização dos sujeitos e com o desenvolvimento de suas próprias estratégias.
\end{abstract}

PALAVRAS CHAVE: TRAJETÓRIAS DE INSERÇÃO TRABALHISTA, JUVENTUDE, INSTITUIÇÕES

\section{A FOCUS ON PROGRAMS FOR THE LABOR INCLUSION OF POOR YOUTH: THE INSTITUTIONAL AS A SUBJECTIVE SUPPORT}

\begin{abstract}
The problems of employment and exclusion of youth have become a true challenge for the public policies and action of the civil society. For a long time, programs and diverse strategies are implemented and that, to a great extent, share an unspecific diagnosis on the background of the problems in the area of employment which are also called «deficits» of formation. These approaches are limited ahead of the complex plot of structural, institutional, familiar, individual and subjective dimensions that place youth, in particular the poorest, in disadvantaged situations. The present article is set out to raise the discussion one of these aspects in particular: the institutional level; in order to reflect on the forms in which the institutions try to take part in the youth transition, facing the processes of individualisation and institutional weakening. In this way, two cases of organizations in Argentina have been selected who set out to generate an intense impact on the subjectivities of these young people, through approache which take into account the «constellations of youth disadvantages», in an ample way. The analysis of two institutions that give preferential rise to the subjective approach, opening the way for reflection on institutional possibilities to provide new collective supports and to contribute to the individualization of the subjects and the development of their own strategies.
\end{abstract}

KEY WORDS: LABOR INSERTION TRAJECTORIES, YOUTH, INSTITUTIONS 


\section{INTRODUCCIÓN}

EL PRESENTE ARTÍCULO SE propone discutir los abordajes institucionales de las intervenciones sociales dirigidas a favorecer la inserción laboral de los jóvenes, poniendo el énfasis del análisis en las dimensiones de formación de habitus, y creación de soportes subjetivos.

Ante la crisis y reconfiguración del mercado de empleo, y el aumento de las desigualdades, la inserción laboral de los jóvenes ha dejado de ser un «estado», observable a través del pasaje de una situación a otra (de la educación al empleo) para pasar a ser un largo proceso de transición laboral, considerado la punta de lanza de procesos macrosociales de transformación global de las sociedades y de los modelos de acumulación. La transición, condicionada estructuralmente, ha comenzado entonces desde hace más de una década a ser objeto de programas sociales orientados a mejorar las oportunidades de los jóvenes, en particular de aquellos con menores niveles educativos y en situación de pobreza.

Medidas centradas especialmente en la capacitación laboral, en las pasantías y en el apoyo a microemprendimientos han intervenido de un modo acotado, fragmentado y con escasa coherencia global e incluso desde perspectivas opuestas (Jacinto, 2008). Se trata, en gran medida, de programas puntuales que muchas veces parten de diagnósticos parciales en torno a las dificultades de los jóvenes en situación de pobreza. En general, comparten un diagnóstico inespecífico sobre el trasfondo de los problemas en el mercado de trabajo y los llamados «déficits» de formación, pero toman escasamente en cuenta dimensiones estructurales, institucionales, familiares, individuales y subjetivas que hacen a la suma de las desventajas juveniles. Entre ellas: el acceso a escuelas de mala calidad, la segmentación territorial que dificulta la salida del barrio, el escaso y deficiente acceso a servicios de salud, el lugar de los jóvenes dentro de sus hogares, la asunción de tempranas responsabilidades, la escasez de oportunidades de formación profesional de calidad y de «puentes» con buenos empleos, las propias perspectivas de los jóvenes sobre el trabajo, etc.

En el marco de una investigación sobre «Trayectorias educativolaborales de jóvenes. Incidencia de políticas y programas de inclusión social», se ha desarrollado un marco analítico que intenta articular las complejas dimensiones estructurales, institucionales, individuales y subjetivas que condicionan el acceso de los jóvenes pobres a buenos 
empleos y plantean desafíos específicos a las estrategias de intervención en este terreno. El presente artículo se plantea discutir en particular uno de ellos: el nivel institucional, examinando en este caso abordajes de instituciones que, como capacitadoras, intentan intervenir en la transición, a la luz de los nuevos desafíos que imponen los procesos de individualización y de debilitamiento institucional de las sociedades contemporáneas, en este caso, la sociedad argentina. De este modo, se han seleccionado dos casos de organizaciones que se proponen generar un fuerte impacto sobre las subjetividades de los jóvenes, a través de abordajes complejos, que toman en cuenta de un modo particularmente amplio, las «constelaciones de desventajas» que padecen los jóvenes que atienden. Con diagnósticos, supuestos, y estrategias diferentes, ambos casos permiten reflexionar sobre las «nuevas institucionalidades» y los soportes subjetivos necesarias para mejorar las oportunidades de los jóvenes.

\section{LA TRANSICIÓN LABORAL DE LOS JÓVENES COMO PROBLEMA SOCIOLÓGICO}

Durante la mayor parte del siglo veinte, la integración de las generaciones jóvenes a la sociedad era imaginada colectivamente como una secuencia de pasos institucionalizados, especialmente canalizada a través de las instituciones educativas y de las ligadas al mundo productivo. Salir de la escuela, desarrollar algún nivel de formación, ingresar en un empleo y construir una carrera profesional a partir de allí eran los hitos de ese proceso. El pasaje a la adultez, entonces, se caracterizó como la salida del hogar de origen, y la asunción de responsabilidades laborales y de reproducción familiar. En este marco, el ingreso al mundo del trabajo ha sido considerado como un momento crucial para la construcción de la identidad social de los individuos y una de las instancias biográficas que muestran privilegiadamente los mecanismos de reproducción y/o cambio social presentes en una determinada sociedad (Galland, 1984). Si durante años determinados ritos y pasajes por distintas instituciones (la escuela, el trabajo, la constitución de una nueva familia...) configuraban las trayectorias de la juventud a la adultez, hoy esos hitos se desdibujan. En lo que concierne al paso al empleo, ingresar al mercado de trabajo no puede considerarse como un «momento» ya que es un largo y complejo proceso hacia un empleo estable, si es que finalmente éste llega (Jacinto, 2007), configurando una transición más o menos larga, más o menos 
calificante. Esta reconfiguracion va dejando nuevas generaciones en situaciones de vulnerabilidad en relación al mercado de trabajo, en particular a los jóvenes provenientes de hogares pobres.

Ahora bien, las formas que asume la transición no sólo están vinculadas a procesos macrosociales y a su correlato sobre características individuales (sector social de origen, el nivel educativo, el género, el capital cultural y social). Dos ejes centrales de la sociedad informacional y del posmodernismo configuran de modos muy diversos esa transición. En particular, el debilitamiento institucional y procesos de individualización condicionan fuertemente las trayectorias de inserción de los jóvenes. El proceso es a la vez biográfico y estructural, e incluye diversas formas de desafiliación, de la educación, del trabajo, pero también de otras formas de sociabilidad que son asimismo mediaciones de reconocimiento social (Castel, 1997). Esta franja de vulnerabilidad se pone especialmente de manifiesto en la organización social de los ciclos de vida, es decir, en las trayectorias escolares, en el pasaje de la educación al trabajo, en las movilidades en el curso de la vida activa, y en el proceso de salida. Estos momentos claves de los procesos de socialización están en crisis desde el doble punto de vista de las instituciones y de los individuos (Dubar, 1996).

La amplia gama de situaciones de vulnerabilidad pone de manifiesto la heterogeneidad de la pobreza, y la ampliación y complejización de las desigualdades en el acceso a recursos y oportunidades, configurando una nueva cuestion social (Castel, 1997). Tal como señalan Fitoussi y Rosanvallon (1997), las nuevas desigualdades ya no están asociadas solamente a las diferenciaciones de orden estructural, sino que proceden de la recalificación de diferencias dentro de categorías a las que antes se juzgaba homogéneas. Los individuos, aún dentro de la misma categoría social no se enfrentan a las mismas situaciones ni conforman un itinerario vital con las mismas características. Una misma cohorte, con las mismas características estructurales, puede contener distintas trayectorias en su interior (Elder, 1994). Los elementos estructurales conforman la matriz de relaciones objetivas por la cual los individuos transitan, pero no explican en su totalidad las particularidades de cada trayectoria.

¿Qué sucede entonces en las etapas de transición laboral de los jóvenes? Abundantes estudios en países centrales y algunos en la Argentina, evidencian hoy una multiplicación de transiciones, de pasajes del empleo al desempleo, y viceversa, del empleo a la inactividad, y aún pasajes del empleo a otro empleo de diferentes condiciones y ni- 
veles de precariedad (Gautié, 2003). En esta compleja trama de diferentes condiciones biográficas, contextos de mercados de empleo locales que brindan oportunidades diferenciadas, acceso a programas públicos de formación-trabajo y a segmentos de empresas diferentes, los estudios de trayectorias han comenzado a evidenciar la creciente diversificación y a señalar una recomposición de las diferentes etapas de la vida activa (Gautié, 2003). Las trayectorias muestran una importante individualización, detectándose variadas formas de articulación entre distintos tipos y grados de educación, capacitación y empleo (Roberts et al., 1994).

Al mismo tiempo las trayectorias no sólo se individualizan sino que también se polarizan. La transición se caracteriza por la diversidad, la inestabilidad y la precariedad de las experiencias laborales y está influida no sólo por la inestabilidad estructural del mercado de empleo sino también por la naturaleza exploratoria de las formas de «ser joven» (Gaude, 1996).

De este modo, los procesos de individualización de las trayectorias de inserción de los jóvenes ponen de manifiesto tanto los condicionantes macroestructurales como las subjetividades en torno, no sólo al trabajo, sino también respecto otras esferas de la vida como personal, familiar y educativa.

La individualización no quiere decir que la estructura social, en términos de origen y oportunidades, haya perdido importancia. La desigualdad social en los recursos y oportunidades persiste también en las trayectorias individualizadas, llevando a opciones biográficas más amplias o más estrechas. La capacidad del individuo de gestionar su propia transición a la vida adulta depende fundamentalmente del capital social, del conocimiento cultural, del apoyo recibido por su familia y las oportunidades o restricciones relativas a la educación, el género, el origen social y étnico (Walther y Pohl, 2005).

Se plantean, entonces, nuevos interrogantes a las políticas sociales que atienden la pobreza y las desigualdades, acerca de cómo traducir las nuevas condiciones en sus diseños e implementaciones.

Algunos esfuerzos por construir miradas que articulen lo macrosocial con los niveles institucionales y subjetivos brindan herramientas teórico-conceptuales sugestivas para el abordaje de la investigación de estos fenómenos, en particular en relación al estudio de las transiciones juveniles y las posibilidades de intervenir en pos de una mayor inclusión social. Adoptaremos en este artículo la perspectiva que sostiene que la transición de los jóvenes con menores oportunidades se 
configura a partir de un interjuego entre las carencias estructurales respecto del acceso, de las formas de gestión y de la relevancia de las oportunidades de transición, y las debilidades en el acceso a recursos a nivel individual (Walther y Phol, 2005). Se configuran, así, «constelaciones de desventajas», entendidas como las complejas relaciones entre los factores socioeconómicos, institucionales e individuales. Las vidas individuales se estructuran según el acceso a recursos y oportunidades, pero también por la forma en que los perciben, interpretan y los gestionan los propios individuos. Desde esta perspectiva, se desprende que las políticas y las intervenciones sociales en pos de la construcción de ciudadanías deben sustentarse en criterios sistémicos, institucionales y subjetivos.

Así como ha comenzado a debatirse sobre las llamadas «políticas de subjetividad» (Tedesco, 2007), y «las políticas en pos de la equidad en las trayectorias» (Rosanvallon, 1995), que plantean acciones flexibles en función de necesidades diferenciadas, plantearemos aquí un análisis empírico y reflexiones acerca del rol de las llamadas «nuevas institucionalidades». Este análisis de dos instituciones que intervienen en la transición de los jóvenes, dando un lugar preferencial a los abordajes subjetivos, abrirá la reflexión acerca de las posibilidades institucionales de proveer nuevos soportes colectivos y contribuir a la individualización de los sujetos y al desarrollo de sus propias estrategias.

\section{LAS NUEVAS INSTITUCIONALIDADES EN LA MEDIACIÓN ENTRE LOS JÓVENES Y SU INSERCIÓN LABORAL}

Desde nuestra perspectiva, la dimensión institucional es central para comprender los alcances de un «dispositivo de inserción» y su peso sobre las subjetividades. Las instituciones constituyen las «mediaciones» entre las políticas o programas sociales y los jóvenes. En este sentido, se evidencia un proceso paradójico en torno al lugar de las instituciones que acompañan o debieran acompañar la transición. Por un lado, se asiste sin duda a la crisis y debilitamiento de las grandes instituciones de integración social de la modernidad. Pero por otro lado, la intervención en el apoyo a la transición requiere tanto el fortalecimiento de las instituciones tradicionales como la construcción de nuevas institucionalidades y nuevos actores institucionales. A continuación fundamentaremos esta perspectiva.

Muchos trabajos recientes han enfatizado en el creciente proceso de «desinstitucionalización», refiriéndose al debilitamiento de los 
soportes institucionales en las biografías individuales. Desinstitucionalización quiere decir que lo que ayer era tomado a cargo colectivamente por las instituciones es ahora transmitido al individuo mismo, quien desde entonces debe asumir, bajo la trayectoria personal, su propio destino (Martuccelli, 2007).

Como ya se ha mencionado, las instituciones de socialización, aquellas cuyo objetivo es accionar directamente sobe las conductas, los sentimientos, los valores y las representaciones de los individuos (Dubet, 1997), que caracterizaron la modernidad y participaban activamente en los procesos de socialización, están cambiando y se debilitan como soportes colectivos. Es precisamente una característica de la modernidad, como ha señalado Dubet (1997), el doble proceso que los marcos institucionales confieren al sujeto: por un lado, la institución socializa al individuo, le imprime un habitus y una identidad conforme a los requisitos de la vida social. Por otra parte, se lo inscribe como un sujeto que pueda constituirse como un actor, dueño de sí mismo, un individuo autónomo. Las instituciones han sido, a lo largo de la modernidad, no sólo dispositivos de control y socialización, sino espacios de subjetivación. Desde una perspectiva centrada en las trayectorias biográficas, las instituciones conforman los soportes organizadores de los tiempos y dinámicas de los recorridos biográficos, al mismo tiempo en que se constituyen en recursos que permiten orientar las estrategias y decisiones en juego en esas trayectorias. Al decir de Beck (2007) las instituciones apoyan al individuo a desarrollar su propia biografía, lo cual no necesariamente debe resaltarse como positivo en cuanto al despliegue de la autonomía individual sino también en el aspecto negativo, de incertidumbre y angustia ante la falta de soportes protectores.

El debilitamiento alcanza a todas las instituciones de socialización. En primer lugar, la familia, con sus múltiples formatos y las tensiones generadas por un contexto desprotector. En segundo lugar, la escuela, otra institución clásica de la modernidad, que se ha visto debilitada como constructora de sentidos y como ámbito de generación de saberes socialmente significativos por varias razones. Estas razones incumben tanto el formato institucional y pedagógico de la escuela, en particular el de la secundaria (Dubet y Martuccelli, 1997). Pero también porque las oportunidades que ofrece no son homogéneas: la configuración de circuitos de diferente calidad, y la construcción social e institucional del fracaso escolar lleva a casi la mitad de los jóvenes argentinos a no terminar la escuela secundaria, y a que los jóvenes pobres que la terminan tengan limitadas oportunidades de «hacer valer» su título (Jacinto, 
2006). Al mismo tiempo, también la inserción en el mundo del trabajo ha perdido seguridad, cediendo su lugar al desempleo, la precarización y la consecuente vulnerabilidad social.

En el contexto argentino se han estudiado los procesos de descolectivización (Svampa, 2005) y fragmentación institucional (Duschatzky y Corea, 2004; Tiramonti, 2004) para señalar los efectos en la conformación de las identidades. Con los patrones institucionales y colectivos de referencia debilitados, los individuos se ven atravesados por contextos de inestabilidad que inciden en la fragmentación y desestructuración de sus recorridos biográficos. Frente a este panorama, los individuos montan sus estrategias vitales por medio de recursos sociales muy desiguales (Martuccelli, 2007). La indeterminación caracteriza muy fuertemente este proceso, y la única certeza que parece quedar es aquella, limitada y modesta, que señala que en el fondo de cada uno es donde hay que encontrar la verdad del propio recorrido identificatorio. Así, las desventajas por las que se atraviesan derivan en una pérdida de la dimensión colectiva; la experiencia individual pasa a ser una empresa privada.

Por otra parte, como ha señalado Bauman (2003), allí donde se debilita la presencia estatal, cobra significación la comunidad local, la comunidad encarnada en un territorio habitado por sus miembros. El territorio, el barrio, pasa a ser el espacio social capaz de inscribir identitariamente a los individuos que allí viven y que conforman un vínculo de semejanza y homogeneidad que se distingue con lo diferente y heterogéneo del entorno exterior. Frente a unas condiciones en donde las instituciones - como el trabajo — han perdido centralidad en los individuos, otras marcas subjetivas parecen ganar vigorosidad. Marcas ligadas al territorio y, sobre todo en el caso de los jóvenes, a los grupos de pares y bandas, y en algunos casos, el paso por nuevas institucionalidades.

También algunas investigaciones que han analizado los nuevos patrones de identificación frente al debilitamiento de los soportes colectivos e institucionales, sostienen que aquellos no se encarnan en las instituciones tradicionales sino en nuevos marcos identitarios ligados a los espacios de residencia y a los grupos de referencia (Merklen, 2005). Estos espacios permiten la construcción de lenguajes y esquemas de percepción que se transforman en recursos — a modo de un capital social— que se hacen necesarios para habitar condiciones de desafiliación.

De acuerdo a estos debates, ¿han dejado de ser las instituciones las mediaciones que permiten encuadrar las trayectorias individuales en procesos sociales más generales? Si se piensa específicamente en 
los jóvenes, es indudable que las instituciones que caracterizaban y garantizaban la integración a la vida adulta se encuentran desestructuradas en relación a su función durante la modernidad. Si hace unas décadas la transición a la vida adulta estaba dispuesta a través de mecanismos institucionales que orientaban el pasaje a la adultez, el contexto actual presenta una profunda desarticulación y fragilidad de estos mecanismos. La ausencia de institucionalidad que pueda hacer un puente con el trabajo no hace más que reforzar mecanismos de reproducción y exclusión social (Jacinto, 2006).

Sin embargo, a pesar de su debilidad, las instituciones por las que atraviesan los jóvenes — si éstas están legitimadas socialmente- se erigen como espacios de socialización. También brindan recursos que, si bien no permiten garantizar los pasajes hacia la adultez, pueden acumularse en términos de capital social y cultural. En efecto, un estudio anterior de trayectorias juveniles (Jacinto, 2006) ha mostrado que la legitimación social de la que goza la institución por la que pasan los jóvenes, brinda redes y puentes con el empleo que de otro modo estarían vedadas. Esta suerte de «transferencia del capital social institucional» resulta muchas veces clave para lograr la inserción.

Aún en un marco de declive institucional, la forma en que se conforman las trayectorias individuales está articulada con los pasajes y experiencias institucionales y es necesario comprender las articulaciones y procesos que éstas despliegan en las biografías individuales. Algunas instituciones tradicionales como las escuelas, aún ante el debilitamiento, se configuran como espacios de inserción social (Jacinto y Freytes Frey, 2004; Tiramonti, 2007) También las instituciones de la sociedad civil cubren un nuevo papel, desde una perspectiva de construcción de lo público no estatal.

En realidad, el lugar de las instituciones tanto en la configuración de las subjetividades como en la construcción de habitus no puede definirse a priori. Actualmente, esos procesos dependen no tanto del tipo de institución sino de cada institución en concreto y de la medida y la forma en que se despliega como espacio de inclusión, de reproducción, o de participación social.

El contexto sociohistórico y estructural es el escenario en que se desarrollan los actores institucionales y las prácticas sociales de los individuos. En los procesos de inserción juega entonces el contexto como condicionante, pero en tanto proceso social también es construido por las políticas y programas orientados a la inserción, por los propios jóvenes, y por las instituciones y organizaciones que son media- 
doras en esa relación entre educación y trabajo.

Las instituciones de la transición intervienen desde varios niveles en la construcción de oportunidades de inclusión social. En el análisis empírico que sigue, examinaremos las perspectivas de dos ONGS, en relación a estos procesos. Analizaremos, en primer lugar, sus perspectivas acerca de la inclusión social en función de sus objetivos, del público al que se dirigen y el mundo del trabajo que avizoran. En segundo lugar, las características que imprimen a la intervención y su contribución a la conformación de subjetividades y de habitus. En tercer lugar, cómo conciben su rol en tanto mediadoras de capital social para los jóvenes. Respondiendo estas preguntas, se espera hacer una contribución a las discusiones acerca del nuevo rol (o el nuevo posible rol) de las instituciones en la construcción de subjetividades y de autonomías y en la generación de habitus.

Los datos empíricos provienen de una investigación en curso sobre trayectorias laborales de jóvenes e incidencia de los dispositivos de inserción laboral. Entre los dispositivos, se han seleccionado programas de formación e inserción laboral de ONGS, dos de las cuales serán examinadas a continuación en la perspectiva de contribuir al análisis conceptual propuesto.

El primer caso, es el programa de formación y trabajo para jóvenes, al que llamaremos el Centro 1. Dicha organización, creada en el año 2005, comenzó sus acciones en la zona sur de la Ciudad de Buenos Aires, implementando un programa cuyo principal objetivo es facilitar la inserción laboral de calidad a jóvenes de segmentos sociales necesitados, en base a un sistema innovador de formación y empleo. El programa ofrece formación a jóvenes que están cursando los últimos años del secundario, en especialidades diseñadas a partir de perfiles de puestos requeridos por el mercado de empleo y asociados a segmentos de «trabajo decente» (según definición de la OIT). Una vez que finaliza la formación, se facilita la inserción laboral de los jóvenes en empresas que ofrecen puestos de calidad, asociadas a una red laboral de empresas conformada por el programa. Durante el siguiente año, una vez que los jóvenes ya se encuentran trabajando, se realizan una tutoría permanente desde la institución.

El segundo caso, que llamaremos Centro 2, está ubicado en Carupá, Municipalidad de Tigre. Se trata de un proyecto dependiente del Obispado y de un Centro de Formación Profesional Provincial, cuyo objetivo principal es la formación laboral de jóvenes de la comunidad y brindarles un espacio de pertenencia, crecimiento y promoción a través de la cons- 
trucción de un proyecto laboral y de vida. El taller brinda cursos de cocina, costura, informática, carpintería, herrería, soldadura, electricidad y tornería. Recibe adolescentes y jóvenes del barrio que hayan cursado o no en el secundario, y/o que se encuentren cursándolo. El Centro fue inaugurado en 1993, por lo tanto lleva 15 años de funcionamiento.

La estrategia metodológica que sustenta este análisis suma etapas sucesivas de conocimiento de estas instituciones. En relación a la más antigua, desde hace varios años se sigue su accionar a través de encuentros con la directora y/o parte del equipo. En el segundo caso, desde el inicio se tuvo oportunidad de entrevistar al equipo a cargo y observar la institución. Además de estos procesos, se han realizado durante septiembre 2008 entrevistas en profundidad a las directivas y se han realizado observaciones no participantes en las instituciones.

\section{a) Las perspectivas sobre la inclusión}

Una cuestión clave acerca del accionar institucional con grupos vulnerables son las concepciones que la sustentan. En particular, es relevante reconocer las perspectivas sobre la inclusión en distintas dimensiones. Una primera dimensión se refiere a cómo conceptualizan la relación entre inserción laboral e inserción social; una segunda a las visiones sobre las problemáticas de la población que atienden y los déficits en cuanto a acceso a recursos y oportunidades, y una tercera dimensión se refiere a las concepciones sobre el mercado de trabajo para el que preparan.

En general, estudios previos de este tipo de programas muestran que esos objetivos suelen convivir con otros más amplios y menos precisos, que apuntan a la integración social de los jóvenes ante la preocupación por la falta de espacios institucionales existentes para aquellos que están desescolarizados, los niveles de violencia y marginación social a las que están sometidos, etc. Algunos estudios cualitativos (Jacinto, 1998, Gallart, 2001, Pieck, 2001) señalan que muchas veces el acceso de los jóvenes vulnerables a alguna instancia de capacitación laboral incide no tanto en el acceso a mejores empleos sino sobre aspectos subjetivos y de trayectorias personales: aumenta su autoestima, facilita el reingreso a la escolaridad, y en algunos casos, las instituciones son percibidas como espacio de protección frente a un contexto social excluyente.

De este modo, inclusión laboral e inclusión social suelen formar parte del enfoque desde el cual se parte. En una primera aproximación, las instituciones examinadas se plantean objetivos de acercamiento al mundo del trabajo, es decir, forman para la empleabilidad y el acceso 
al empleo. Sin embargo, las concepciones y los énfasis tienen sus matices. El modo en que ambas instituciones se plantean la inclusión social y laboral, es diferente, como se observa en los siguientes testimonios:

El objetivo principal de la Fundación es darles a los chicos un salto de calidad, para que puedan acceder a un buen primer empleo de calidad una vez que egresan de la escuela secundaria. Agregarles valor a través de una formación, que les va a servir para su posterior inserción. El valor es lo humanístico; lo técnico, es la excusa. El valor más fuerte de esta organización es hacer una diferencia en la calidad de vida de esta población. Y eso no quiere decir tal o cual profesión, sino cómo se va a desempeñar en esa profesión. Nosotros aspiramos a incluir al 100\% de los chicos que formamos en empleos de calidad. El día que no podamos garantizar eso, tendremos que generar una nueva especialidad [...]. Hay buenas empresas. Hay buenos empleadores. Poner a estos pibes en un entorno donde los cuidan y les exigen a la vez, es una gloria (Centro 1).

Nosotros queremos abrirles oportunidades a los chicos, que puedan verse de otra manera, mostrarles nichos de oportunidad. Es una toma de conciencia en general: de su ciudadanía, de sus derechos. Para nosotros lo fundamental es que cambien su actitud hacia el trabajo. Que asocien su proyecto laboral a un proyecto de vida. Los chicos que vienen acá no tienen al trabajo como un valor (Centro 2).

Entonces mientras en una de las instituciones aparece el énfasis en la inclusión laboral de calidad, en la otra, desde un abordaje territorial que atiende sectores más pobres, el énfasis está puesto en brindarles una experiencia subjetiva que lleve a los jóvenes a reconocer el trabajo como un valor.

Una de las razones por las cuales las perspectivas sobre la inserción social difieren es que ambas instituciones atienden poblaciones de perfiles bien diferentes. Mientras que el Centro 1 define su población a partir de ciertas características individuales de los jóvenes (esencialmente estudiantes secundarios pobres), el Centro 2 lo hace a partir de una perspectiva territorial: se dirige a todos los jóvenes del barrio donde está inserto.

En efecto, el Centro 1 se enfoca hacia «chicos pobres, que están estudiando en el secundario, pero no con chicos marginales», planteando que su intervención se dirige principalmente a dos objetivos que reflejan su diagnóstico acerca de los recursos y oportunidades de 
los jóvenes. Un objetivo es suplir déficit de formación en aptitudes y conocimientos para el trabajo motivados por las debilidades del sistema educativo formal. Otro objetivo se propone intervenir respecto a dificultades para el acceso de estos jóvenes a empleos de calidad: debido a que no cuentan con redes familiares y capital cultural y social que les permita acceder a un buen empleo una vez que terminan el secundario, el título de nivel secundario está lejos de garantizar una trayectoria laboral de calidad. Así lo expresan:

Todavía está el concepto de que si llegaste al secundario completo te salvaste, y no es verdad. Es cierto que un título ayuda, es una credencial que te habilita. Pero es una condición no determinante. Esto no se le muestra a los chicos En zona sur, cuando los chicos egresan, si no logran insertarse en buenos empleos, terminan teniendo trayectorias laborales más precarias (Centro 1 ).

En el Centro 2, las debilidades respecto a la escuela secundaria no sólo se refieren a su calidad, sino también al propio acceso. Teniendo en cuenta las características del barrio donde está ubicado y la falta de escuelas secundarias en la zona, el Centro se ha posicionado desde su inicio como un complemento del secundario pero también como una alternativa al mismo. De este modo, la intervención se concibe de un modo integral: formar jóvenes para el trabajo, pero también compensar saberes básicos (se volverá sobre estos aspectos) y constituirse en un espacio de pertenencia, alternativo de «estar en la calle». En coherencia con este planteo, la inclusión es entendida como un proceso amplio, permitiendo el ingreso de todos los jóvenes que se presentan, más allá de características particulares.

Recibimos chicos del barrio, es un barrio con muchas carencias. Recibimos chicos que están en la escuela y que no están. [...] Nosotros tomamos a todos los chicos. Se hace una entrevista inicial, pero entran todos avisándoles que acá se hace de todo. Se viene a la carpintería, pero además, se va a ver matemática, se va a hablar de la droga. [...] Los chicos que después no les va la dinámica, dejan. O sea, la selección no la hacemos nosotros, va decantando solo (Centro 2).

Una tercera dimensión acerca de las perspectivas sobre la inclusión se refiere al mercado de trabajo para el que forman. Los estudios sobre enfoques de los programas de formación para el trabajo dirigidos a jóvenes con escasas oportunidades han mostrado que las intervenciones suelen ir 
desde aquellas que enfatizan la inserción en el empleo, muy cercanas a los requerimientos de determinados sectores o nichos del mercado de trabajo, hasta instituciones que repiten cursos tradicionales año a año, independientemente de las posibilidades concretas o potenciales de inserción en el mercado de trabajo. En los programas de carácter social, se han observado cursos orientados al sector informal y/o al autoempleo, muchas veces de escasa calidad y pocos recursos (Jacinto, 2008).

Los casos que se están examinando en este artículo se distinguen de esos enfoques, porque garantizan la posibilidad de brindar oportunidades de ingreso a empleos de calidad. Sin embargo, en función de los diferentes condicionantes estructurales que viven las poblaciones que atienden como de los propios recursos, las perspectivas institucionales varían. ¿Hacia qué mercado forma uno y otro Centro? El Centro 1 lo define a partir del empleo protegido, en blanco. No sólo forma, sino que desarrolla estrategias explícitas para insertar a los jóvenes en esos empleos, cuestión que se presenta como un gran desafío.

Les proponemos pasar a un empleo donde existan como empleados, que tenga obra social, aportes, que esté en blanco.

¿Por qué nosotros ofrecemos curso de camarero o vendedor? Porque seguimos al mercado laboral. Lo que demanda el mercado, allá vamos. Yo entrevisté más de 200 empresas para buscar el nicho de demanda de egresados de secundario que les falta un cachito de formación. Te digo que es buscar una aguja en un pajar. Por un lado está el tema de que el secundario es un requisito básico para muchísimos empleos de calidad. En ciertos circuitos, se pide más formación, como por ejemplo inglés (Centro 1).

En el relevamiento de empresas, el enfoque es bien de mercado. El enfoque no es «beneficiemos a chicos pobres». Es el opuesto: «vení, que vas a ser el mejor». Y con las empresas es lo mismo, «yo te puedo brindar lo que necesitás». Nuestro enfoque está puesto en el mercado (Centro 1).

En el Centro 2, se asume cierta impotencia frente a las distancias entre las exigencias del mercado laboral y los perfiles de los jóvenes que atiende, y señala que las oportunidades de inserción de estos jóvenes suelen darse en trabajos informales o en la economía social. No es que no se reconozcan empleos de mayor calidad, sino que aparece como un mundo lejano a las posibilidades de los jóvenes del barrio.

Toda la situación económica y social, fue para nosotros un desafío. No- 
sotros hace 15 años que estamos y fuimos repensando los cursos de acuerdo a lo que se iba demandando. Costura en los noventa estuvo parada y ahora se reactivó. Por ejemplo ahora, tenemos el curso de informática [...]. Nosotros estamos atentos a qué cosas se están pidiendo desde afuera en el mercado de trabajo. La inserción laboral formal es muy difícil para los chicos que salen de los cursos. La situación laboral, el mercado es muy exigente y reducida, para gente que viene con las competencias básicas muy débiles. Sí hay oportunidades en todo lo que es la economía social, los microcréditos (Centro 2).

A pesar de este panorama general, han logrado que algunos jóvenes accedan a puestos en circuitos de calidad. Pero en esta institución se plantean incidir en un aspecto más amplio respecto al trabajo, sosteniendo que promueven la «cultura del trabajo», que se ha visto fuertemente afectada tanto por el desempleo como por la irrupción masiva de planes sociales.

Nosotros vemos que la «cultura del plan» como la llamamos nosotros, incidió muchísimo en lo que es la cultura del trabajo. El otro día, un chico de herrería, para trabajar con nosotros, decía «¿pero me van a blanquear?». «Primero vas a estar un tiempo a prueba y después sí, por supuesto». «¿Pero entonces me van a sacar del plan?». «¿Pero cuánto ganás con el plan, 150 pesos? Acá ganás 800. ¿Qué te parece?». «Sí, pero después ¿y si no me va?». Y el no me va quiere decir: no me lo banco, llego tarde, quiero faltar. No saben cuánto van a aguantar despertarse temprano.

Con las familias hay que trabajar también, porque vienen y te dicen «falta porque tiene que cuidar a los hermanitos». Luchamos con los papás para que valoren esto, porque el chico tiene que responsabilizarse, comprometerse con lo que está haciendo. No es lo mismo que venga o no venga. Hacemos un esfuerzo por incidir también en la cultura del barrio, donde está tan debilitado lo que es lo formal y la cultura del trabajo (Centro 2).

Los casos reflejan dos concepciones acerca de la inclusión que, sin embargo, se asemejan en el compromiso general de ofrecer mayores oportunidades a los jóvenes. Consideran que deben intervenir comprometida y explícitamente como instituciones mediadoras, para crear puentes con mundos que parecen distantes de los jóvenes que atienden. Ahora bien, los condicionantes estructurales, la pobreza de los hogares, las debilidades de las instituciones previas por las que pasaron los jóvenes, en particular la escuela como así también la segregación territorial, son distintos y de mayor gravedad en un caso que en el 
otro y limita fuerte y diferentemente los márgenes de acción. Ambas se proponen de este modo incidir en los habitus de los jóvenes y en el proceso de socialización laboral.

\section{b) La personalización de la intervención}

Se ha señalado en la primera parte del texto que los procesos de individualización de trayectorias apelan cada vez más al desarrollo de políticas sociales que apunten a las subjetividades. Muchas dimensiones de la inclusión social y laboral aparecen vinculadas a la subjetividad: autoestima, valores, actitudes, motivaciones, expectativas.

Algunos estudios sobre programas que actúan en la capacitación laboral de jóvenes muestran persistentemente que las intervenciones más amplias están lejos de constituir un mero curso de formación ocupacional. Componentes como orientación sociolaboral y personal, fortalecimiento de saberes y competencias básicas, y acompañamiento posterior al curso son cada vez más incluidos en las versiones más abarcativas de este tipo de programas sociales (Jacinto, 2006).

Un alto grado de personalización, de seguimiento cuidadoso, distingue las dos instituciones estudiadas, aunque como se ha visto, se diferencian en las perspectivas en que abordan la inclusión. Las actitudes de los jóvenes, recuperar la autoestima, estimular la comunicación oral resultan los focos privilegiados de su accionar. Trabajar con las subjetividades está en el centro de la estrategia.

Lo que aporta este programa es netamente actitudinal: la honestidad, la responsabilidad. Lo técnico es una excusa (Centro 1).

El aprendizaje de los chicos es más que nada subjetivo, descubrir otra forma de relacionarse con el trabajo, revalorizar sus saberes, trabajar la autoestima de los chicos. Son chicos estigmatizados, desvalorizados por todo su entorno, por la escuela, pero también por la casa (Centro 2).

Un énfasis particular aparece con la estimulación de la comunicación oral de parte de los jóvenes, ya que esta cuestión refleja un obstáculo para la inserción laboral y social.

Del lado de los pibes, hay una sensación que no es para ellos, que no pueden, es terrible la introversión de los pibes. Pibes con la gorrita que no te miran a los ojos. Lugares que no sienten para ellos. Yo no sé que es lo que hace la escuela en relación a la extroversión, pero yo creo que 
influye que las actividades hoy son más solitarias, se han perdido los vínculos cara a cara. Los pibes están sentados todo el día frente a una computadora. Se perdieron las instancias de exposición. Antes nos tomaban lección, pasábamos al frente. Un pibe de 18 años, ¿de dónde saca la extroversión cuando va a un primer trabajo? En una entrevista lo que te están evaluando ¡es cómo hablás! Entonces, acá hay mucho por hacer. Trabajamos mucho la cuestión de la comunicación, permanentemente. A través de los role playing, pero también la comunicación más técnica, el vocabulario, como pararse frente a un público. Trabajamos con una fonoaudióloga (Centro 1).

Hay un idioma cultural diferente, de poca expresión y demás. Que va mejorando a medida que entran en confianza y que se van apropiando de lo que van haciendo. Hay que trabajar mucho el tema de la violencia también. Estamos hablando de chicos que en general son expulsados de las instituciones educativas, que vienen de situaciones muy traumáticas. Entonces, todo lo que es institucional, al principio cuesta (Centro 2).

El abordaje de la subjetividad se produce a través de estrategias altamente personalizadas. En ambas instituciones, se observa un énfasis puesto en el acompañamiento permanente. Las acciones no pueden reducirse a la formación, sino que tienen que estar acompañadas por otras acciones, tales como la tutoría permanente, la recreación y la orientación de los jóvenes.

Estamos constantemente marcándoles cosas, reflexionando con ellos sobre sus aspiraciones, sus experiencias. Además, tienen una tutoría de un año que nos permite acompañarlos durante la experiencia laboral después de la formación (Centro 1).

Acá se lo reconoce a cada uno. Es un trato muy personalizado. Se trabaja la cuestión vincular. Hacemos entrevistas iniciales y de seguimiento, además se trabaja con los padres [...]. Los padres dicen «vengo a la reunión de acá, y es distinta a la de la escuela». Claro, porque trabajamos temáticas que les interesan, rescatamos a sus chicos siempre desde lo positivo [...]. Hay mucha apropiación. Mucho compromiso y apropiación desde el principio (Centro 2).

Nosotros en el 98 instalamos esta parte de orientación laboral. Porque veíamos que un pibe se capacitaba en un oficio, pero después no sabía cómo salir del barrio, tomarse un colectivo y buscar trabajo. Así, empezamos a incorporar este espacio que trabajamos desde lo que es un trabajo para mí, hasta información sobre el mercado laboral, orientación, 
armado de currículum, entrevista laboral (Centro 2).

De este modo, ambas instituciones se transforman en fuertes lugares de pertenencia y referencia para los jóvenes, adonde los jóvenes regresan una y otra vez aunque hayan finalizado su formación:

La motivación más importante por la cual los chicos permanecen en los cursos es el lugar de referencia, la pertenencia [...]. Los que más aprovechan la experiencia son los adolescentes, por la continuidad. Porque vuelven y vuelven, por una cuestión de lo que se ofrece como institución [...]. Todos tienen la puerta abierta acá. Los chicos que tienen que preparar un trabajo para la escuela y les prestamos los computadores, las mujeres que están trabajando en costura y se les rompe la máquina, vienen a usar éstas. Todos saben que en el Taller les vamos a dar una mano (Centro 2).

Sin embargo, la concepción acerca de qué actitudes desarrollar en los jóvenes varían de acuerdo a los objetivos y al contexto en se desenvuelve cada institución. Mientras el Centro 1 aspira a generar un espacio lo más similar posible al ámbito laboral, para que los jóvenes en ese marco adquieran las aptitudes necesarias para desenvolverse en un empleo, el Centro 2 pretende brindar a los jóvenes formación en un sentido más amplio y al mismo tiempo más básico. Así en el Centro 1 presentan del siguiente modo su estrategia:

Nuestra fórmula es 50\% dureza, $50 \%$ afecto. Porque ¿cómo trata un jefe a un empleado? lo trata con afecto, lo guía con firmeza y lo desafía a superarse. Eso es un buen jefe y de esa misma manera queremos relacionarnos con los chicos. Lo que les falta a los chicos hoy es personas que los guíen con firmeza. Se perdió el valor de la organización, del respeto por la autoridad. Después cuando van a laburar, al jefe lo tratan como a un igual y a los dos días no están trabajando más. [...] Si hay algo en lo que yo no voy a transigir nunca es en subestimar a los pibes, porque después los pibes creen que no les da la cabeza. No estás determinado por venir de donde venís. La apuesta está en la capacidad de construir, elegir la vida que uno quiere y no en la cultura de la queja.

En cambio, en el Centro 2 parten del reconocimiento del débil punto de partida, para ir proponiendo un acompañamiento personalizado y largo:

Acá pueden aprender un montón de cosas, pero nosotros sabemos que los chicos vienen con un déficit estructural enorme, que no se repara en un año. A veces vemos que los chicos hacen trayectorias más largas acá en la 
institución: primero arrancan con carpintería, después hacen soldadura y terminan en computación. Y vos ves cómo recién después de tres años, los chicos se acomodan, se les abre la cabeza, y ahí empiezan con el secundario o lo terminan. Empiezan a estructurarse de otra manera.

Una certeza empieza a consolidarse al examinar estos testimonios. Estas instituciones de la sociedad civil se erigen como espacios sustantivos de construcción de las subjetividades de estos jóvenes, cubriendo un lugar en dónde la escuela secundaria parece ausente. ¿Cuánto pesan estas experiencias en las trayectorias de estos jóvenes? ¿Reparan y aportan a la inclusión social? Lejos de tener un carácter instrumental en relación a la inserción laboral, tal vez la incidencia más relevante de este tipo de experiencias sea contribuir a procesos mucho más amplios de subjetivación (Dávila, 2003, Jacinto, Abdala y Solla, 2005). Sólo observando las trayectorias posteriores de los jóvenes puede responderse esta cuestión; cuestión que se estudiará en la siguiente etapa de la investigación. Sin embargo, otros estudios previos de este tipo de experiencias suelen señalar alta gratificación de los jóvenes con el pasaje por este tipo de experiencias y reconocimiento de aprendizajes que no hubieran realizado en otros espacios (Jacinto, 2008).

\section{c) La institución como mediadora de capital social}

La inserción de un joven en un empleo no sólo está vinculada a una compleja trama que vincula los condicionantes estructurales, el valor de los títulos, conocimientos y competencias, las estrategias de gestión de las empresas, etc., sino también a las puertas de acceso a los empleos. En particular, muchas investigaciones muestran que las redes de relaciones sociales tienen un fuerte peso sobre las oportunidades de inserción (entre otros, Weller, 2006, Moreno, Gallart y Cerruti, 1992; Jacinto 1996).

Tal vez uno de los mayores aportes a las trayectorias de los jóvenes de los programas de intervención social sea el fenómeno que hemos denominado «la transferencia de un capital social institucional» (Jacinto, 1996). Es conocido que el capital social, entendido como red de relaciones sociales y también como asociado a un capital cultural (o sea conocimientos, ideas, valores y habilidades), resulta uno de los mecanismos de reproducción social más poderosos: intervenir en la ampliación de ese capital puede ser entonces crucial en la expansión de las oportunidades. En los casos analizados esta transferencia apare- 
ce de muchas maneras, señalamos en particular dos: las instituciones se transforman en «puentes con los empleos» y su prestigio institucional pasa a ser reconocido como un atributo de los propios jóvenes.

$\mathrm{El}$ «puente con el empleo» se materializa en las experiencias analizadas, en la medida en que ambos centros se orientan a facilitar el acceso de los jóvenes a un circuito de inserción laboral al que no tendrían acceso por sus propias redes de relaciones. Como se ha visto, uno de ellos trabaja por insertar a los jóvenes en empleos «decentes» y lo hace a través de la gestión directa en las empresas con las que establece acuerdos, acompañando a los jóvenes durante un año. El otro, desde su perspectiva territorial, ha generado varios emprendimientos, abre sus puertas para que el vecindario pueda utilizar sus recursos, etc. De este modo, está contribuyendo a que la comunidad pueda establecer los «vínculos débiles» que según la formulación de Granovetter, (1973) son fuertes e indispensables para las oportunidades individuales y para su integración en las comunidades. Como se ha sostenido con anterioridad (Jacinto y Bessega, 2002), estos puentes que logran quebrar circuitos cerrados muestran la importancia de que las intervenciones no se conciban simplemente como el acceso a un conocimiento valioso, útil y/o significativo, sino también con una clara conceptualización de los obstáculos que operan en los mecanismos de selectividad del mercado de trabajo y el aporte que puede hacer la institución en tanto mediadora en ese sentido.

Del mismo modo, el otro sentido en que la institución transfiere su capital social es constituyéndose en un lugar de referencia para los empleadores y/o para la comunidad.

Lo que nosotros hacemos es brindarles todo lo que brinda una experiencia de un buen trabajo. Eso lo da el trabajo naturalmente; la Fundación aspira a brindárselos a los chicos que de por sí solos no tendrían la posibilidad de acceder a ese empleo. [...] La idea es que cada vez más empresas nos digan «queremos chicos de la Fundación porque son diferentes. A eso apuntamos» (Centro 1).

Aspiramos a trabajar un lenguaje institucional diferente, donde la puerta está abierta pero no se escapan... Algunas empresas nos dicen «prefiero tomar a los chicos del Taller», porque saben que acá se trabaja lo actitudinal y eso hace la diferencia (Centro 2).

De este modo, estarían operando tanto un capital social vertical como horizontal. Tal como sostiene Dávila (2003), existe un nivel de capital social «instalado en las instituciones» y otro en el nivel de relaciones más 
cercanas, y ambos son reconocibles en los dos casos estudiados. Coincidimos con este mismo autor en que ambos pueden contribuir al desarrollo de las subjetividades y de los proyectos juveniles. Aunque deberían estar asociadas a una estructura de oportunidades más amplias.

\section{REFLEXIONES FINALES}

Los casos estudiados sorprenden: más allá de sus amplias diferencias respecto al público que atienden y al mercado de trabajo en que avizoran insertar a sus jóvenes, se parecen en aspectos claves vinculados a la formación de habitus, a la socialización y a la afirmación de las subjetividades. Cada uno desde su punto de partida, se propone hacer aportes sustantivos en estos sentidos. Aunque será en el seguimiento de las trayectorias de los egresados donde pueda sostenerse con fundamento cual ha sido la incidencia, los abordajes muestran un aporte sustantivo a la inclusión social de los jóvenes.

Sin duda, la contribución al capital social tanto relacional como transferido puede ampliar sustantivamente las oportunidades, especialmente en relación a las primeras experiencias de empleo. Pero ello aparece claramente diferenciado entre una y otra institución, ya que tanto los objetivos institucionales como las distancias de los jóvenes atendidos con empleos de calidad son bien distintas.

El Centro 1 aspira claramente a intervenir sobre los obstáculos de los jóvenes pobres que terminan el secundario para acceder a empleos de calidad. Entre los obstáculos ese Centro conceptualiza dos cuestiones claves: la debilidad de los aprendizajes realizados en las escuelas secundarias a las que accedieron y la falta de un capital social que facilite el acceso a un empleo de calidad. No es un tema menor intervenir en la formación actitudinal y en el puente con el empleo, ya que las estadísticas muestran que para ellos, las oportunidades de estar desempleados son mayores que para los no pobres que no terminaron el secundario y también las posibilidades de acceder a empleos de calidad son menores respecto a ese grupo.

En el Centro 2, se enfatizan otros ejes, dentro de la perspectiva que ofrece un barrio segregado, donde ni siquiera se cuenta con escuela secundaria. Principalmente, se enfatiza en el fortalecimiento de una cultura del trabajo, es decir, una socialización respecto a qué es trabajar y por qué contribuye a la formación integral del sujeto. La percepción de los destinos laborales es más difusa, aunque se realizan acciones que intentan abrir puertas tanto con empleos de calidad como con 
emprendimientos colectivos. Así como el primer Centro enfatiza la individualización y el hacerse cargo de la propia estrategia, este segundo Centro pone en foco la solidaridad grupal, barrial como instancias de inclusión social y laboral. Una pregunta sin duda se instala: ¿hasta dónde puede llegar la inclusión social en el marco de condicionantes estructurales tan fuertes? Esta pregunta remite a otra más general ¿es posible tender puentes durables más allá del segmento social al que pertenecen los jóvenes atendidos?

También es particularmente importante la forma en que estas experiencias apuntan a intervenir sobre las motivaciones de los jóvenes. Tanto desde el sentido del trabajo y del esfuerzo, como desde la ampliación de las expectativas: acceder a un empleo de calidad, seguir estudiando, terminar el secundario forman parte discursos y estrategias explícitas. En este sentido, abren mundos de los que los jóvenes antes no sólo estaban excluidos, sino que también habían interiorizado como «para los otros». «Eso no es para mí, yo no puedo» son las palabras que repetidamente mencionan los jóvenes en sus primeros acercamientos a estas instituciones.

De este modo, las perspectivas de estas instituciones llevan a reflexionar tanto sobre la importancia de sus intervenciones en tanto soportes organizadores de las trayectorias como en cuanto a los recursos que se proponen brindar para orientar estrategias y decisiones de los jóvenes.

Al menos dos fuentes de reflexión se abren sobre otras instituciones que intervienen en la transición laboral de los jóvenes: ¿Qué podría una escuela secundaria aprender de estas prácticas? ¿En qué medida las políticas sociales y de empleo que apuntan a la inserción de jóvenes pobres conceptualizan estas complejidades, en cuanto a los abordajes institucionales que parecen ser necesarios (aunque no necesariamente suficientes) para mejorar las oportunidades de inclusión de los jóvenes? Ambos interrogantes pueden, sin duda, contribuir a profundizar la reflexión sobre la dimensión institucional de las políticas de inserción laboral dirigidas a los jóvenes.

BuENo Aires (ARgENTINA), DICIEMBRE DE 2009

RECIBIDO: DICIEMBRE 2008

ACEPTADO: MARZO 2009 
Inclusión laboral de los jóvenes pobres

\section{REFERENCIAS BIBLIOGRÁFICAS}

BAUMAN, ZYGMUNT (2003): Comunidad. En busca de seguridad en un mundo hostil. Buenos Aires: Siglo XXI.

BECK, UlRICH y ELISABETH BECK-GERNSCHEIM (2007): La individualización. El individualismo institucionalizado y sus consecuencias sociales. Buenos Aires: Paidós.

CASTEL, ROBERT (1997): La metamorfosis de la cuestión social. Una crónica del salariado. Buenos Aires: Paidós.

DÁVILA, OsCAR et al. (2003): Capital social juvenil y evaluación programática hacia jóvenes. Santiago: Instituto Nacional de la Juventud.

Dubar, Claude (1996) : «Socialisation et processus». En Serge Paugam: L'exclusion: l'état des savoirs. Paris: Editions La Découverte.

Dubet, François (1997): El declive de la institución. Profesiones, sujetos e individuos ante la reforma del Estado. Buenos Aires: Gedisa.

— y DANilo MARTUCCELLI (1997): En la escuela. Sociología de la experiencia escolar. Buenos Aires: Losada.

DUSCHATZKY, SILVIA y CRISTINA COREA (2002): Chicos en banda. Los caminos de la subjetividad en el declive de las instituciones: Buenos Aires: Paidós.

ELDER, GLEN (1994): «Time, human agency and social change: perspectives on the life course». Social Psychology Quarterly, vol. 57, №1.

Fitoussi, JeAn PAUl y PierRe Rosanvallon (1997): La nueva era de las desigualdades. Buenos Aires: Manantial.

GALLAND, OlivieR (1984): «Precarité et entrées dans la vie ». Revue Française de Sociologie XXV, Paris.

GALLART, MARÍA ANTONIA (2001): «Los desafíos de la integración social de los jóvenes pobres: la respuesta de los programas de formación en América Latina». En ENRIQUE PIECK (coordinador): Los jóvenes y el trabajo. México: Cinterfor.

- MARTín MoReno y MARCELA CeRruti (1993): «Educación y empleo en el Gran Buenos Aires 1980-1991. Situación y perspectivas de investigación». Cuadernos del CENEP Nº49. Buenos Aires: CENEP.

GAUDE, J. (1996): «Relations entre nouvelles formes de travail, la formation et l'insertion professionnelle». Etudes de Politique N²3. Geneve: OIT.

GAUTIE, JeRome (2003): «Transitions et trajectoires sur le marché du travail». Quatre pages No59. Paris: Centre d'études de l'emploi.

— (2000): «Inserción profesional y desocupación de los jóvenes en Francia». Revista Argentina de Economía y Ciencias Sociales, Vol. IV, №6. Buenos Aires: Ediciones de la Universidad.

GRANOVETTER, MARK (1973): «The strength of weak ties». American Journal of Sociology, Vol. 78, $\mathrm{N}^{\circ} 6$.

JACINTO, ClAUDIA (2008): «Políticas públicas, trayectorias y subjetividades 
en torno a la transición laboral de los jóvenes». En PÉREz Sosto (editor): El Estado y la reconfiguración de las protecciones sociales. Buenos Aires: Instituto Torcuato Di Tella y Siglo XXI Editores.

(2006): «Estrategias sistémicas y subjetivas de transición laboral de los jóvenes en Argentina. El papel de los dispositivos de formación para el empleo». Revista de Educación N³41. Madrid: MEC.

- (1996): «Desempleo y transición educación-trabajo en jóvenes de bajos niveles educativos. De la problemática estructural a la construcción de trayectorias». Dialógica Vol. 1, número especial. Buenos Aires: CEIL.

— y MARÍA ANTONIA GALlART (1998): Por una segunda oportunidad: la formación para el trabajo de jóvenes vulnerables. Montevideo: Cinterfor.

— y CARLA BESSEgA (2002): «Un lugar en el mundo. Jóvenes vulnerables en búsqueda de espacios de inclusión social». En FlOREAL FORNI (compilador): De la exclusión a la organización. Hacia la integración de los pobres en los nuevos barrios del conurbano bonaerense. Buenos Aires: Ediciones Ciccus.

— y AdA FREYTES FREY (2004): Políticas y estrategias para el mejoramiento de las oportunidades de los jóvenes: estudio sobre la educación secundaria en la Ciudad de Buenos Aires. París: IIPE-UNESCO.

__ Ernesto Abdala y Alejandra Solla (2005): La inclusión laboral de los jóvenes: entre la desesperanza y la construcción colectiva. Montevideo: Cinterfor.

—-, Alejandra Solla, Cristina García y Ana SuÁrez (2007): «Jóvenes vulnerables y programas sociales: lógicas desencontradas, abordajes acotados». $8^{\circ}$ Congreso de ASET, Buenos Aires.

MARTUCCELli, DANILO (2007): Gramática del individuo. Buenos Aires: Losada. MERKLEN, DENIS (2005): Pobres ciudadanos. Las clases populares en la era democrática (Argentina 1983-2003). Buenos Aires: Ediciones Gorla.

PIECK, ENRIQUE (2001): Los jóvenes y el trabajo. La educación frente a la exclusión social. México: Coedición UIA, IMJ, UNICEF, CINTERFOR-OIT, RET Y CONALEP.

ROBERTS, KENNETH; STAN CLARK y Claire WALLACE (1994): «Flexibility and individualisation: a comparison of transitions into employment in England and Germany». Sociology Journal of the British Sociological Association, Vol. 28, $\mathrm{N}^{\circ} 1$.

ROSANVALLON, PIERRE (1995): La nueva cuestión social. Buenos Aires: Nueva Visión.

SVAmpa, Maristella (2005): La sociedad excluyente. La Argentina bajo el signo del neoliberalismo. Buenos Aires: Taurus.

TEDEsco, JuAn CARLos (2007): «Democracia y educación». Conferencia Magistral dictada en el $44^{\circ}$ curso de rectores. Buenos Aires: CONSUDEC.

TiRAMONTI, GUILLERMinA (2007): «Nuevos formatos escolares para promover la inclusión educativa. Un estudio de caso: la experiencia argenti- 
na». Documento de FLACSO. Buenos Aires: FLACSO.

(2004): «La configuración fragmentada del sistema educativo argentino». Cuadernos de Pedagogía N¹2. Rosario: Centro de Estudios de Pedagogía Crítica.

WALTHER, ANDREAS y AXEL PHOL (2005): Thematic study on policy measures concerning disadvantaged youth. Tubingen: Iris.

WELLER, JÜRGEN (2006): «Inserción laboral de jóvenes: expectativas, demanda laboral y trayectorias». Boletín Redetis N²2. Buenos Aires: Redetis. 